Reactor design and integration with product detection to accelerate screening of electrocatalysts for carbon dioxide reduction

Ryan J. R. Jones, Yu Wang, Yungchieh Lai, Aniketa Shinde, and John M. Gregoire

Citation: Review of Scientific Instruments 89, 124102 (2018); doi: 10.1063/1.5049704

View online: https://doi.org/10.1063/1.5049704

View Table of Contents: http://aip.scitation.org/toc/rsi/89/12

Published by the American Institute of Physics

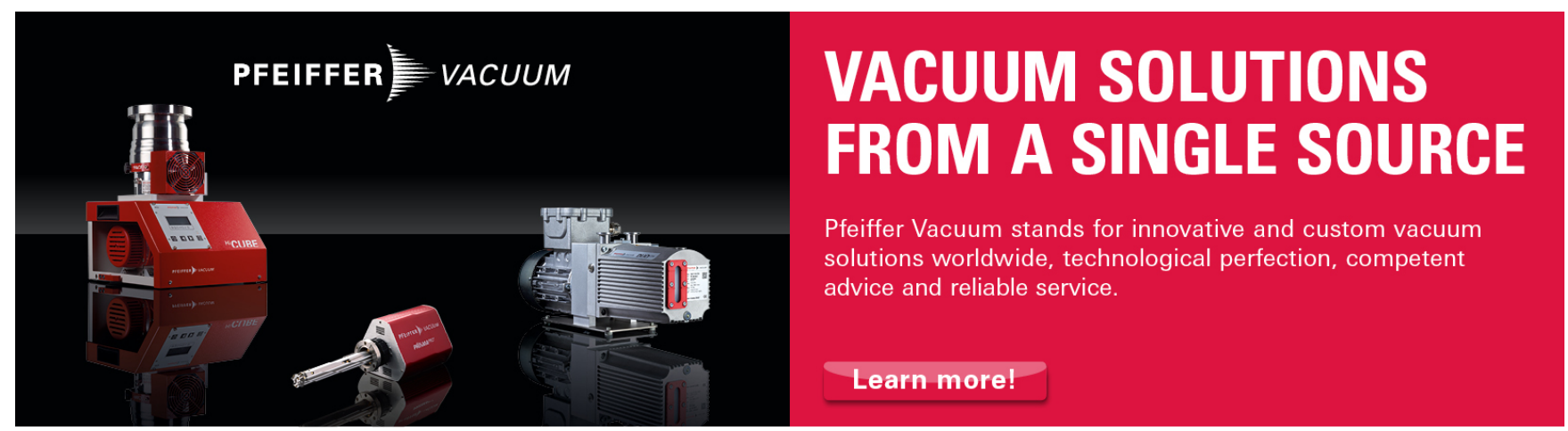




\title{
Reactor design and integration with product detection to accelerate screening of electrocatalysts for carbon dioxide reduction
}

\author{
Ryan J. R. Jones, Yu Wang, Yungchieh Lai, Aniketa Shinde, and John M. Gregoirea) \\ Joint Center for Artificial Photosynthesis, California Institute of Technology, Pasadena, California 91125, USA
}

(Received 24 July 2018; accepted 2 December 2018; published online 26 December 2018)

\begin{abstract}
Identifying new catalyst materials for complex reactions such as the electrochemical reduction of $\mathrm{CO}_{2}$ poses substantial instrumentation challenges due to the need to integrate reactor control with electrochemical and analytical instrumentation. Performing accelerated screening to enable exploration of a broad span of catalyst materials poses additional challenges due to the long time scales associated with accumulation of reaction products and the detection of the reaction products with traditional separation-based analytical methods. The catalyst screening techniques that have been reported for combinatorial studies of (photo)electrocatalysts do not meet the needs of $\mathrm{CO}_{2}$ reduction catalyst research, prompting our development of a new electrochemical cell design and its integration to gas and liquid chromatography instruments. To enable rapid chromatography measurements while maintaining sensitivity to minor products, the electrochemical cell features low electrolyte and head space volumes compared to the catalyst surface area. Additionally, the cell is operated as a batch reactor with electrolyte recirculation to rapidly concentrate reaction products, which serves the present needs for rapidly detecting minor products and has additional implications for enabling product separations in industrial $\mathrm{CO}_{2}$ electrolysis systems. To maintain near-saturation of $\mathrm{CO}_{2}$ in aqueous electrolytes, we employ electrolyte nebulization through a $\mathrm{CO}_{2}$-rich headspace, achieving similar gas-liquid equilibration as vigorous $\mathrm{CO}_{2}$ bubbling but without gas flow. The instrument is demonstrated with a series of electrochemical experiments on an Au-Pd combinatorial library, revealing non-monotonic variations in product distribution with respect to catalyst composition. The highly integrated analytical electrochemistry system is engineered to enable automation for rapid catalyst screening as well as deployment for a broad range of electrochemical reactions where product distribution is critical to the assessment of catalyst performance. Published by AIP Publishing. https://doi.org/10.1063/1.5049704
\end{abstract}

\section{INTRODUCTION}

Extensive resources have been allocated toward determining the feasibility of devices that create or convert solar or electrical energy into chemicals for efficient storage, transport, and on-demand use. Solar fuel generators are one such promising technology which utilize sunlight to drive electrochemical reactions to convert inexpensive and readily available feedstocks into fuels. ${ }^{1,2} \mathrm{CO}_{2}$ reduction to compressible and/or liquid fuels is particularly attractive, yet solar or electrochemical generation of carbon-containing fuels has been hindered by poor efficiency and selectivity of the catalysts reported to date, prompting the need to search for new catalysts. ${ }^{3-7}$ Here we present a material characterization instrument designed to quantitatively evaluate the efficiency and selectivity of novel catalysts for the direct conversion of carbon dioxide into transportation fuels under conditions commensurate with commercially viable solar fuel generators.

Combinatorial techniques are particularly well suited for carrying out catalyst searches in a basic research setting, as mapping the composition-structure-performance relationship of catalytic materials provides fundamental insights into their function. ${ }^{8-10}$ Just as a catalyst's performance specifications will differ from application to application, so will the

a)gregoire@caltech.edu instruments and techniques used to characterize the catalyst for its intended purpose. Catalyst characterization ideally involves operation of the catalyst in conditions relevant to the desired application, with subsequent transport of the liquid and/or gaseous products to an analytical instrument for identification and quantification of reaction products. These methods are defined by and are unique to the desired application, yet three primary components are common to electrocatalyst screening: an electrochemical reaction vessel, a sampling mechanism, and an analytical tool. In general, the depth of information provided by the analytical tool is inversely related to the breadth of samples probed per unit time; e.g., speed vs. accuracy trade-offs are common, motivating design of high throughput screening techniques that strike the appropriate balance for accelerating catalyst discovery.

Traditional H-style cells are the most common reaction vessels for electrocatalyst screening because of their penchant for enabling highly detailed and precise characterization. An H-cell typically consists of two gas-tight compartments separated by an ion exchange membrane: the cathodic and anodic compartments. In some configurations, carrier gas is continuously delivered into the cathodic compartment and the effluent stream is vented directly into a gas phase analysis instrument. In others, the batch catholyte is sampled by using a syringe at regular intervals or at the end of the experiment for accumulated liquid product analysis. The analytical instrument used for $\mathrm{H}$-cell characterization is 
dependent on the reaction products of interest. Gas Chromatography (GC) is most commonly used to analyze a wide range of permanent gas/organics/hydrocarbons with a reasonable analysis time (3-60 min/sample). ${ }^{11-13}$ Other analytical instruments including High Performance Liquid Chromatography (HPLC), ${ }^{14,15}$ nuclear magnetic resonance (NMR) spectroscopy, ${ }^{11}$ ion chromatography, ${ }^{16}$ and ultraviolet-visible (UV-Vis) spectroscopy ${ }^{17}$ were reported for liquid phase analysis with sample throughput rates commonly reported at $<10$ samples/day.

Kuhl et al. ${ }^{3}$ recently developed a reaction vessel based on large planar electrodes with small electrolyte volume to accelerate concentration of products and enable detailed analytical chemistry information for the $\mathrm{CO}_{2}$ reduction reaction $\left(\mathrm{CO}_{2} \mathrm{RR}\right)$. By combining online $\mathrm{GC}$ for gas phase and ex-situ NMR for liquid phase analysis, they were able to discover new reaction products with extremely low partial current density (i.e., $<0.01 \mathrm{~mA} / \mathrm{cm}^{2}$ ) over copper catalysts. With both this type of cell design and H-type cells coupled with analytical devices, the cell design and operation are typically focused on providing in-depth analytical information regarding product distribution with the breadth of samples investigated limited by the long time product accumulation and slow product analysis. ${ }^{11}$

Differential electrochemical mass spectrometry (DEMS) is an analytical technique that combines an electrochemical reactor with a mass spectrometer. ${ }^{18-20}$ It has been widely utilized to provide "real-time" detection of electrochemical reactants/products/intermediates. However, common issues associated with DEMS include the need for extensive electrode preparation,$^{21}$ the influence of mass transport at the working electrode surface, and a complicated cell configuration. ${ }^{22,23}$ Online electrochemical mass spectrometry (OLEMS) limits the impact of these issues while retaining the advantage of real-time detection. OLEMS features an MS inlet which consists of a tiny capillary tip covered ${ }^{24}$ or embedded with a PTFE membrane. ${ }^{25}$ This tip inlet is placed near the working electrode $(10-50 \mu \mathrm{m})$ to implement direct sampling of the reaction surface. Capillary inlets are also employed to create multielectrode scanning DEMS (SDEMS) cells for investigating electrode arrays/libraries of different types and compositions ${ }^{26,27}$ however, depending on the position the capillary inlet is placed (i.e., how close it is to the electrode surface), OLEMS and SDEMS will only "locally" sample reaction products and create an opportunity for the analytical instrument's response to be poorly representative of the global electrocatalyst performance. This type of localized product detection can be particularly troublesome for characterizing $\mathrm{CO}_{2} \mathrm{RR}$ catalysts due to the importance of product selectivity and the possibility that the product distribution varies over the working electrode (WE) area.

A variety of scanning flow-cells have been developed to enable the rapid collection of electrochemical information for the characterization of both discrete ${ }^{28}$ and continuous ${ }^{29}$ composition libraries. Klemm et al. ${ }^{30}$ further studied corrosion processes by characterizing material dissolution via a scanning flow cell (SFC) coupled to inductively coupled plasma mass spectrometry (ICP-MS), enabling a throughput of $\sim 10^{2}$ samples per day. Their SFC was later coupled to OLEMS for characterization of volatile reaction products. ${ }^{31}$ Using this approach, they demonstrated semi-quantitative evaluation of the electrochemical production rate of hydrogen and some $\mathrm{CO}_{2} \mathrm{RR}$ products on polycrystalline copper surfaces, with sample throughput limited by the recovery time of the analytical tool and Faradaic efficiency (FE) quantification limited by the use of mass spectroscopy instead of separation-based detection. The authors further note concerns with electrochemical conditions and collection efficiency in the OLEMS-coupled device, including the issue noted above that only a small fraction of the electrode area is sensed by using the analytical probe. ${ }^{32}$ To address these concerns, Clark et al. ${ }^{32}$ designed a DEMS cell to fully quantify all reaction products from $\mathrm{CO}_{2} \mathrm{RR}$ analytically. It is an excellent tool for a detailed study of select catalysts, but similar to several cells noted above, it is not amenable for broader catalyst screening due to resource intensive electrode preparation. To complement this suite of existing electrocatalyst characterization platforms, we developed a high throughput screening instrument that maintains excellent control over reactor operation and analytical characterization of reaction products with the following design criteria related to $\mathrm{CO}_{2} \mathrm{RR}$ catalyst screening: (1) rapid quantification of typical $\mathrm{CO}_{2} \mathrm{RR}$ products with a $\mathrm{FE}$ detectability limit of approximately $1 \%$; (2) accelerated measurement throughput by coupling electrochemical and analytical chemistry methods with comparable operation time scales; and (3) mitigation of variations in mass transport and electric potentials across the working electrode surface to facilitate translation from catalyst screening to device implementation. Details of experiment operation are included in Sec. II with the design, operation, and demonstration of the $\mathrm{CO}_{2} \mathrm{RR}$ catalyst screening system described in Sec. III.

\section{EXPERIMENTAL}

\section{A. Pd-Au thin film catalyst synthesis}

To demonstrate the operation of the high-throughput analytical electrochemistry system, a Pd-Au composition spread library was fabricated using DC magnetron co-sputtering of $\mathrm{Pd}$ and $\mathrm{Au}$ metal targets at 6 mTorr Ar pressure onto a $100 \mathrm{~mm}$-diameter $\mathrm{Si}$ wafer with an approximately $170 \mathrm{~nm} \mathrm{SiO}{ }_{2}$ diffusion barrier, using a previously described sputter system with $10^{-5}$ Pa base pressure. ${ }^{33}$ The composition gradients in the co-sputtered continuous composition spreads were attained by positioning the deposition sources in a non-confocal geometry. The deposition proceeded for $15 \mathrm{~min}$ with the power on the $\mathrm{Pd}$ and Au sources at 50 and $54 \mathrm{~W}$, respectively, with no additional substrate heating. Similar depositions of pure Pd and pure $\mathrm{Au}$ films were performed using each sputter source individually, providing the pure element compositions of the Pd-Au combinatorial spread. After deposition, the films were stored in a nitrogen purge box to mitigate oxidation or contamination from air exposure.

\section{B. Product detection}

The gas chromatography (GC, Thermo Scientific ${ }^{\mathrm{TM}}$ TRACETM 1300) is equipped with a capillary TG-BONDQ column and a capillary CarboPLOT column. The former column is connected with a split/splitless injector (SSL) and a 
flame ionization detector (FID) as the front GC route. With helium as carrier gas (2 SCCM), this route is applied to analyze methane, ethane, ethylene, higher hydrocarbons up to $\mathrm{C}_{4}$, and liquid products including methanol, acetaldehyde, ethanol, propionaldehyde, acetone, 2-propanol, allyl alcohol, and 1-propanol (the order of liquid product is based on the retention time). The latter column is connected with another SSL and a thermal conductivity detector (TCD) as the rear GC route. With argon as carrier gas (20 SCCM), this route can analyze hydrogen, air, carbon monoxide, and carbon dioxide. An earlier configuration, which was used in the present work for experiments with Cu foil, used a TG-BONDQ column (instead of CarboPLOT) for the rear route with helium carrier gas at 2 SCCM to detect carbon monoxide and carbon dioxide; this configuration was not used in Pd-Au experiments due to the insensitivity to $\mathrm{H}_{2}$. Prior to a day's experiments, $\mathrm{GC}$ gas phase calibration is conducted by purging the cell with calibration gas and performing the following procedure to simulate operational sampling and injection.

At the end of each electrolysis, the gaseous products concentrated in the cell headspace are sampled by using a robotic sample handling system (RSHS, CTC Analytics) and injected into front and rear injectors in a serial fashion. Each gaseous sample aliquot, one for the front and one for the rear injector, is $500 \mu \mathrm{L}$. The oven temperature was held at $110^{\circ} \mathrm{C}$ for both $\mathrm{GC}$ routes. The duration for separation is $10 \mathrm{~min}$. The liquid catholyte is subsequently sampled $(0.8 \mu \mathrm{L})$ and injected into the front GC route (helium as carrier gas with a flow rate of 2 SCCM) with the oven held at $150{ }^{\circ} \mathrm{C}$. The duration for separation is $8 \mathrm{~min}$. A liner with glass wool is located in the GC injector to trap the salt injected, so only the evaporated liquid can enter the GC column for separation. Furthermore, the amount of salt in each injection is as small as $0.01 \mathrm{mg}$ per injection (i.e., calculated by the concentration of bicarbonate used in this study), enabling hundreds of injections before cleaning or replacement. For $\mathrm{Cu}$ experiments, the gas sample aliquot is $250 \mu \mathrm{L}$ for the front injector and $100 \mu \mathrm{L}$ for the rear injector. The liquid sample aliquot is $0.8 \mu \mathrm{L}$ for the front $\mathrm{GC}$ injector. The temperature program was identical for both gas and liquid analysis: it was held at $60^{\circ} \mathrm{C}$ for $2 \mathrm{~min}$, ramped at $90^{\circ} \mathrm{C} / \mathrm{min}$ to $150^{\circ} \mathrm{C}$ and held for $3 \mathrm{~min}$, and finally ramped at $50^{\circ} \mathrm{C} / \mathrm{min}$ to $200^{\circ} \mathrm{C}$ and held for $2 \mathrm{~min}$.

Liquid products are also analyzed via $10 \mu \mathrm{L}$ catholyte injections into a HPLC (Thermo Scientific UltiMate 3000), equipped with an Aminex HPX 87H column (Bio-Rad) and RefractoMax 521 Refractive Index (RI) detector (Thermo Scientific). The $30 \mathrm{~min}$ separation proceeds at an oven temperature of $60{ }^{\circ} \mathrm{C}$ with $0.6 \mathrm{~mL} / \mathrm{min}$ flow of $1 \mathrm{mM} \mathrm{H}_{2} \mathrm{SO}_{4}$ as eluent. The HPLC was calibrated for formic acid, acetic acid, oxalic acid, glyoxal, glycolaldehyde, ethanol, and propanol. The RI detection method provides reasonable detection limits for all of these products, although research focused on a subset of these products may benefit from a different or additional detection technique, such as ultraviolet absorption or mass spectrometry.

\section{Electrochemistry}

Electrolysis was carried out with a Gamry Reference $600^{\mathrm{TM}}$ potentiostat. All electrochemical data were collected using a $\mathrm{Ag} / \mathrm{AgCl}$ reference electrode (LF2, Innovative Instruments) and converted to a reversible hydrogen electrode (RHE) scale using the measured solution $\mathrm{pH}$. The uncompensated solution resistance was measured by performing electrochemical impedance spectroscopy (EIS) in the frequency range of $100 \mathrm{~Hz}-500 \mathrm{kHz}$ with an amplitude of $10 \mathrm{mV}$ at the open circuit potential of a Pt-Pt working electrode-counter electrode system. The uncompensated resistance, $R_{u}$, was measured by using a Nyquist plot of the EIS spectra and was found to be $72 \Omega$. Prior to the electrolysis, the electrolyte $\left(0.08 \mathrm{M} \mathrm{KHCO}_{3}+0.02 \mathrm{M} \mathrm{CsHCO}_{3}\right)$ [potassium bicarbonate ( $>=99.95 \%$ trace metals basis), cesium bicarbonate $(99.9 \%$, Sigma Aldrich)] was purged with $\mathrm{CO}_{2}(99.999 \%$, Airgas) for at least $30 \mathrm{~min}$. Cesium bicarbonate was added to the electrolyte due to cation size playing a significant role in $\mathrm{CO}_{2} \mathrm{RR}$, i.e., small cations favor hydrogen evolution reaction (HER), whereas large cations, e.g., $\mathrm{Cs}^{+}$, favor $\mathrm{CO}_{2} \mathrm{RR}$ and suppress HER. ${ }^{34}$ A bipolar membrane (Fumasep ${ }^{\circledR}$ FBM single film, Fumatech) was used to separate the working and counter electrodes. Platinum wire (99.9\%, Sigma Aldrich) was used as the counter electrode. The surface area of the counter electrode was about $0.25 \mathrm{~cm}^{2}$, while the working electrode surface area was $0.32 \mathrm{~cm}^{2}$.

Hydrogen intercalation is a well-known phenomenon for Pd and its alloys $;{ }^{35}$ therefore, 1 min chronopotentiometry (CP) at $-3 \mathrm{~mA} / \mathrm{cm}^{2}$ was performed until hydrogen intercalation reached equilibrium for Pd containing catalysts. For each composition of the library, 4 sequential electrolyses were performed: (a) $15 \mathrm{~min} \mathrm{CP}$ at $-3 \mathrm{~mA} / \mathrm{cm}^{2}$, (b) $15 \mathrm{~min} \mathrm{CP}$ at $-1 \mathrm{~mA} / \mathrm{cm}^{2}$, (c) $4.5 \mathrm{~min} \mathrm{CP}$ at $-10 \mathrm{~mA} / \mathrm{cm}^{2}$, and (d) $15 \mathrm{~min} \mathrm{CP}$ at $-3 \mathrm{~mA} / \mathrm{cm}^{2}$. The final electrolysis is a duplicate of the first to assess any drift in catalyst performance and/or instrumentation during the sequence of electrolyses. At the end of each electrolysis, gaseous and liquid products were sampled by the RSHS and analyzed by GC (liquid and gaseous product) and HPLC (liquid product). The cell and all solution handling lines are purged with fresh electrolyte and $\mathrm{CO}_{2}$ between electrolyses to avoid cross-contamination.

\section{RESULTS AND DISCUSSION}

\section{A. High throughput analytical electrochemistry (HT-ANEC) instrumentation}

While rapidly quantifying all major gaseous and liquid products is key to identifying good catalysts, quantifying minor products can provide additional insight into the fundamental reaction mechanisms taking place, ${ }^{3}$ and a high throughput instrument therefore demands a compromise between analytical precision and sample throughput. The configuration of the HT-ANEC system is illustrated in Fig. 1, where the hardware integration is designed to also enable software integration. The HT-ANEC software platform is under development and is beyond the scope of the present report focusing on the instrument's analytical capabilities and physical operation. A key design principle for high throughput operation is the establishment of commensurate time scales for both electrochemistry and analytical chemistry to eliminate periods of non-operation that create operational bottlenecks. 


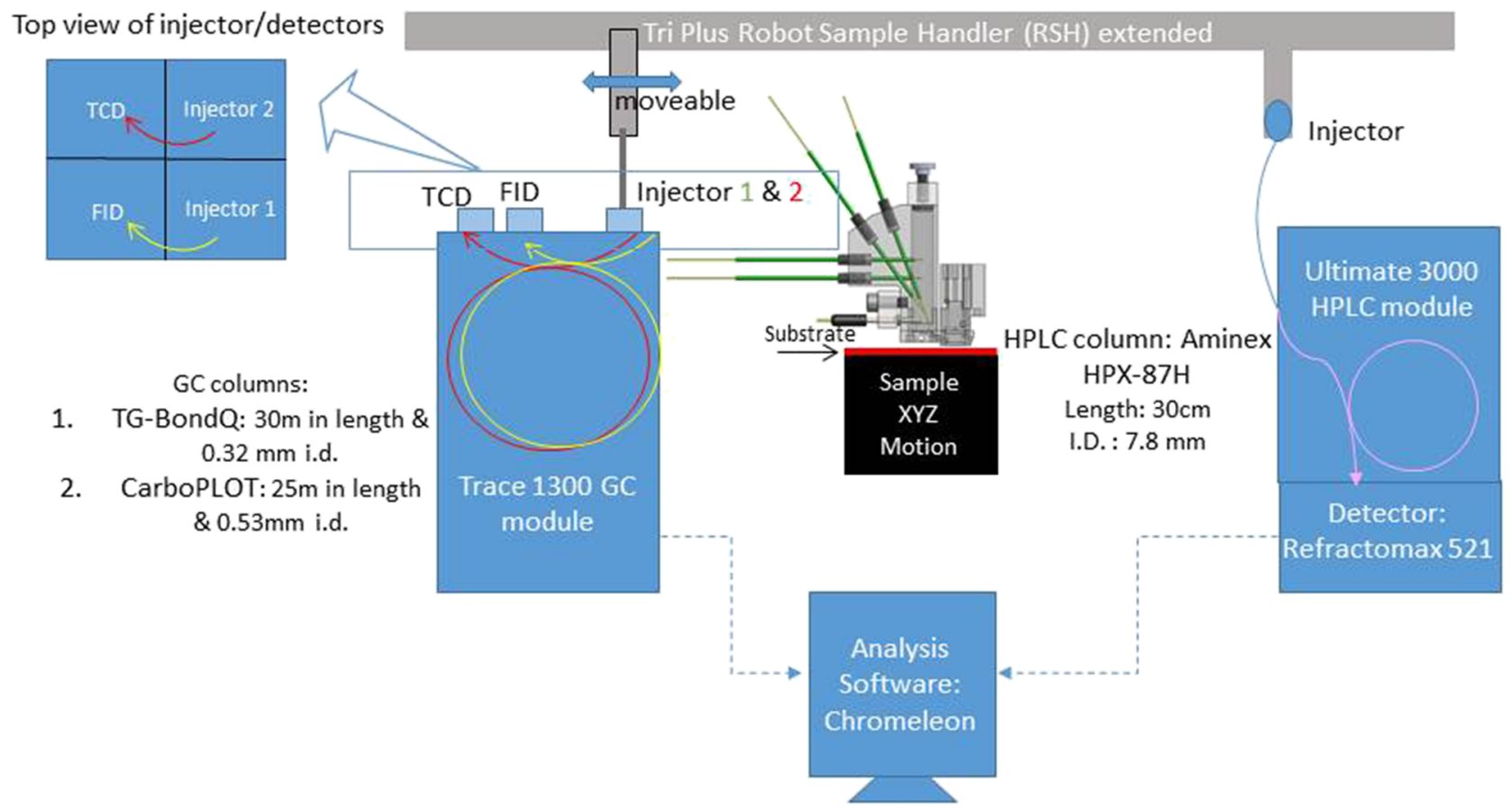

FIG. 1. HT-ANEC schematic showing analytical instrumentation integrated via robotic solution handling to the electrochemical reactor ("REC Cell"). Integrated operation involves sealing the REC cell to the substrate and then performing an electrolysis on a target sample, during which all products are contained within the electrolyte recirculation chamber. Upon completion of the electrolysis, the RSH physically samples the REC cell via syringe penetration of a rubber septum. The liquid, or gaseous, samples are finally transported to their respective analytical instrumentation for analysis via the Chromeleon chromatography data system.

As described below, the 10.5 min duration of the GC separations guided the design of an electrochemical reactor that, within a few minutes, provides $\mathrm{CO}_{2} \mathrm{RR}$ product concentrations which meet the desired $\sim 1 \%$ FE detectability limits.

\section{B. Recirculating electrochemical cell (REC) design and operation}

Electrolysis efficiency and product selectivity from the electrochemical reduction of $\mathrm{CO}_{2}$ to valuable fuels have been shown to depend highly on the system level design of the vessel used for the reaction. Weekes et al. ${ }^{36}$ concluded that flow-cell style reactors are more amenable to commercial applications than traditional $\mathrm{H}$-cell style reactors due to commensurate mass transport and should be considered when designing testing platforms for electrocatalytic materials. Furthermore, Kuhl et al. ${ }^{3}$ have shown that the product efficiency and selectivity of $\mathrm{CO}_{2} \mathrm{RR}$ on $\mathrm{Cu}$ are highly dependent on the applied potential and that the working and counter electrodes should be placed in a parallel geometry to minimize the electric potential variation across the catalyst surface. Ion exchange membranes that separate the catholyte and anolyte chambers also mitigate parasitic reactions from taking place, in particular, oxidation of $\mathrm{CO}_{2} \mathrm{RR}$ products at the counter electrode and oxygen reduction reaction (ORR) at the working electrode.

Traditional $\mathrm{CO}_{2} \mathrm{RR}$ reactor designs ensure sufficient $\mathrm{CO}_{2}$ delivery to the electrode surface by rapidly bubbling gaseous $\mathrm{CO}_{2}$ through the electrolyte; however, dilution of reaction products with electrolyte or headspace flow poses challenges for analytical chemistry. This prompts the design of a recirculating cell that recycles the electrolyte while maintaining rapid solution flow over the WE. Nebulizing, or spraying, drops of solution in a sealed gaseous headspace introduces liquid-gas interfaces similar to that of bubbling the gas in a liquid electrolyte. This "reverse bubbling" action allows for enrichment of the $\mathrm{CO}_{2}$ depleted catholyte stream with a fixed headspace, as opposed to traditional bubbling approaches where rapid flow of gaseous $\mathrm{CO}_{2}$ dilutes the effluent $\mathrm{CO}_{2} \mathrm{RR}$ products. This recirculation approach further decreases the electrolysis time necessary to produce product concentrations necessary for product detection by rapid accumulation in a fixed-volume headspace. A custom recirculating electrochemical cell (REC), and the necessary solution handling to automate reactor operation, was developed to incorporate these design aspects and is shown in Fig. 2. This design allows variation of the headspace volume, electrolyte volume, and electrode area to suite specific research needs, with this implementation of REC using $3 \mathrm{~mL}$, $1 \mathrm{~mL}$, and $0.32 \mathrm{~cm}^{2}$, respectively. The surface to volume ratios ultimately determine throughput due to the need to produce concentrated reaction products for rapid quantification, and the headspace volume places a limit on maximum electrolysis time, as described in detail below.

The three component reaction vessel, which consists of the working electrode (WE) chamber, the counter electrode (CE) chamber, and the electrolyte recirculation chamber, is machined from polyether ether ketone (PEEK) and is sealed with Viton O-rings. The working electrode chamber seals to the catalyst on one side and an ion exchange membrane on the opposing side. The same ion exchange membrane is simultaneously sealed to the CE chamber opposite the WE chamber and ensures that the catholyte and anolyte are segregated during experimentation. The $\mathrm{CE}$ is positioned parallel and in 


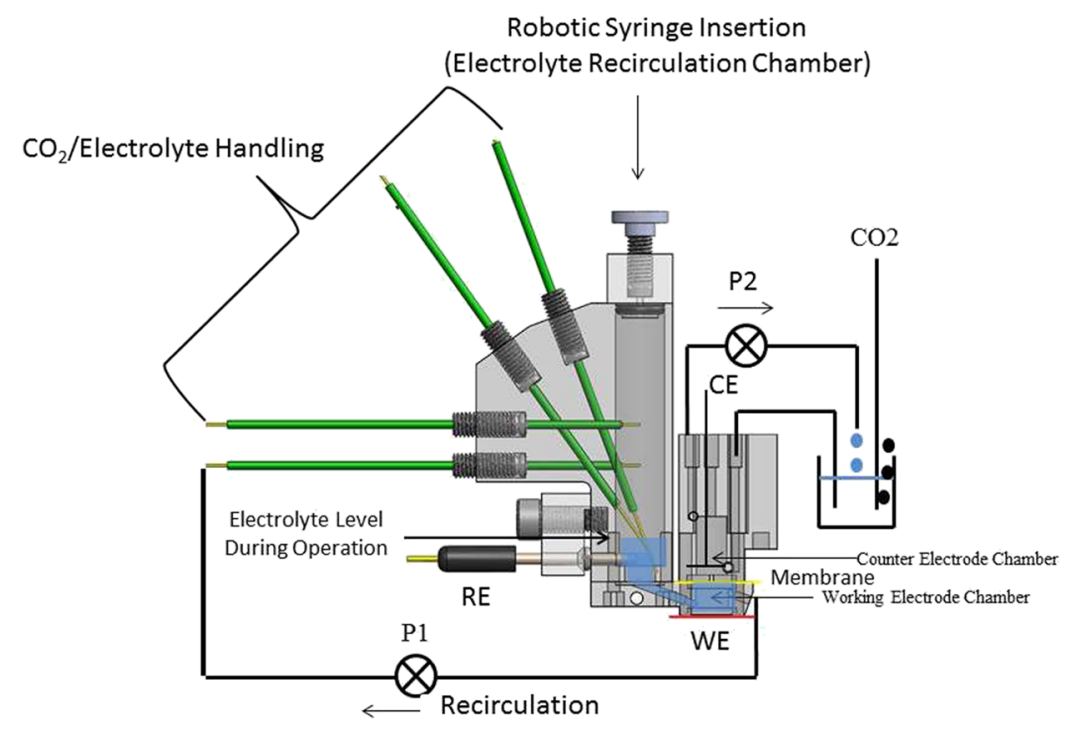

FIG. 2. Recirculating Electrolysis Cell (REC) reactor for controlled $\mathrm{CO}_{2} \mathrm{RR}$ experimentation. The catholyte is highlighted in blue and is recirculated with the pump P1 from the working electrode chamber to the electrolyte recirculation chamber, which contains the static headspace and sufficient electrolyte to enable syringe sampling for product analysis through the noted septumsealed syringe insertion point. The anolyte is recirculated with the pump P2, but with no need for product detection from this chamber, the solution reservoir is not sealed and is bubbled with $\mathrm{CO}_{2}$. close proximity to the membrane, without making contact, approximately $6 \mathrm{~mm}$ from the working electrode. A peristaltic pump ensures that any gas products, for example, evolved oxygen from the anodic CE reaction, are evacuated from the $\mathrm{CE}$ chamber by recirculation with a larger external reservoir continuously sparged with $\mathrm{CO}_{2}$.

The WE chamber is further coupled to an electrolyte recirculation chamber to mitigate variations in the electrolyte composition during operation. A reference electrode resides in the electrolyte recirculation chamber and enables 3-electrode measurements to be made via the conductive solution recirculation pathway that connects to the WE chamber. As noted previously, commercially viable solar fuel generators will likely take advantage of flowing-electrolyte paradigms in order to manage feedstock $\mathrm{CO}_{2}$ concentrations and to concentrate products that accumulate at the catalyst surface to enable economical separation of desirable products. With this motivation, during the execution of an electrochemical profile, a peristaltic pump directs the catholyte from the electrolyte recirculation chamber to the working electrode surface, then from the working electrode surface to the membrane to achieve reliable wetting/contact, and finally back to the electrolyte recirculation chamber where the gaseous products are concentrated. The return pathway to the recirculation chamber terminates in a micro-capillary tube of inner diameter $0.2 \mathrm{~mm}$ which is perpendicular to a planar feature machined into the body of the electrolyte recirculation chamber. The capillary tubing and planar feature effectively nebulize the catholyte stream into the $\sim 1$ atm $\mathrm{CO}_{2}$ headspace of the electrolyte recirculation chamber to restore $\mathrm{CO}_{2}$ in the electrolyte following the $\mathrm{CO}_{2} \mathrm{RR}$ in the WE chamber and to evaporate gaseous $\mathrm{CO}_{2} \mathrm{RR}$ products dissolved in the electrolyte. This action also has the effect of sweeping products away from the catalyst surface to prevent supersaturation and bubble formation from influencing the local catalyst environment. The liquid products (ionic species or molecules with large Henry's constant) mostly remain in the electrolyte, which at sub- $1 \%$ concentrations do not strongly alter the electrolyte composition as the electrolyte is recirculated to the WE chamber to support the continued $\mathrm{CO}_{2}$ electroreduction.
The assembly is mechanically secured in space to a spring loaded holder for repeatable sealing to a planar substrate with a fixed force of approximately $10 \mathrm{~N}$. The cell is coupled to analytical tools via the RSHS. This allows a planar substrate containing a combinatorial catalyst library to raster beneath the REC via computer controlled XYZ motion stages (Dover, FM series) and allow ingress to the electrolyte chamber via rubber septum for the robotically controlled syringe of the RSHS to enable gas and liquid sampling. By allowing the RSHS to alternate between different syringe types (gas vs liquid) and volumes, the sampling of the headspace and electrolyte can be tailored to the analytical tools and their specific operation to provide the desired analytical data for the research project at hand.

The electrolyte recirculation chamber is sealed with a rubber septum to allow for product sampling by serial robotic syringe insertions. While the sealed cell offers substantial benefits, it also introduces variability in electrochemical conditions over time, requiring design of operational parameters to mitigate those variations. The depletion of $\mathrm{CO}_{2}$ from the sealed headspace corresponds to a decrease in the partial pressure of $\mathrm{CO}_{2}$, which shifts the equilibrium with the bicarbonate electrolyte and thus the equilibrium $\mathrm{pH}$ of the electrolyte. By limiting the $\mathrm{CO}_{2}$ consumption to $5 \%$ of the initial $\mathrm{CO}_{2}$ in the headspace, the partial pressure change from $1 \mathrm{~atm}$ to $0.95 \mathrm{~atm}$ corresponds to an equilibrium $\mathrm{pH}$ shift from 6.8 to 6.84 that corresponds to a Nernstian shift of $2.4 \mathrm{mV}$ vs SHE, which is negligible for catalyst screening purposes. This limit on $\mathrm{CO}_{2}$ consumption corresponds to a limit on electrolysis charge, which we calculated using a conservative estimate that assumes the maximum $\mathrm{CO}_{2}$ consumption per electrolysis charge, i.e., $0.5 \mathrm{CO}_{2}$ molecules per electron for $100 \% \mathrm{FE}$ to $\mathrm{CO}$ and/or formate. The resulting maximum allowed total electrolysis charge is proportional to the headspace volume, and by designing the REC cell to have $3 \mathrm{~mL}$ headspace volume, $1.18 \mathrm{C}$ of $\mathrm{CO}_{2} \mathrm{RR}$ charge can pass while maintaining a sufficiently constant $\mathrm{CO}_{2}$ partial pressure. This maximum charge sets the maximum electrolysis duration for a given current density; e.g., at $3 \mathrm{~mA} / \mathrm{cm}^{2}$ with a $0.32 \mathrm{~cm}^{2}$ electrode, the maximum electrolysis time per the quasi-static conditions is 
TABLE I. The Faradaic Efficiency (FE) detection limits for analytical instruments coupled to the REC cell showing limits for a sealed headspace recirculation scheme as used in HT-ANEC operation and with conventional $\mathrm{CO}_{2}$ gas bubbling and electrolyte flow-through schemes. The detection limit was estimated by dividing the minimum GC or HPLC-detectability quantity of product (using the noted injection volumes) by the electrolysis charge (i.e., $5 \mathrm{~min}$ at $3 \mathrm{~mA} / \mathrm{cm}^{2}$ ), providing the minimum FE from this electrolysis that would be detectable. The HT-ANEC system provides excellent detection limits for all typical $\mathrm{CO}_{2} \mathrm{RR}$ products, $\sim 30 \times$ improvement over traditional reactor operation. For products detectable by both GC and HPLC, the better detectability limit is shown. For example, EtOH has an HPLC detection limit of $0.5 \mathrm{mM}$, corresponding to a FE detection limit of $68 \%$, which offers no advantage over the limit provided by the GC.

\begin{tabular}{|c|c|c|c|c|}
\hline \multirow[b]{2}{*}{$\begin{array}{l}\mathrm{CO} 2 \mathrm{RR} \\
\text { products }\end{array}$} & \multicolumn{4}{|c|}{ FE detection limits for 5 min electrolysis at $3 \mathrm{~mA} / \mathrm{cm}^{2}$} \\
\hline & $\begin{array}{l}\text { GC with sealed headspace } \\
\text { in REC cell }(\%)\end{array}$ & $\begin{array}{c}\mathrm{GC} \text { with } 20 \mathrm{SCCM} \mathrm{CO}_{2} \\
\text { flow-through headspace }(\%)\end{array}$ & $\begin{array}{l}\text { HPLC with } 9.5 \mathrm{~mL} \mathrm{~min}^{-1} \\
\text { recirculation in the REC cell }(\%)\end{array}$ & $\begin{array}{l}\text { HPLC with } 9.5 \mathrm{~mL} \mathrm{~min}{ }^{-1} \text { electrolyte } \\
\text { flow-through without recirculation (\%) }\end{array}$ \\
\hline Hydrogen & 0.84 & 28 & & \\
\hline Carbon monoxide & 2.51 & 85 & & \\
\hline Formate & & & 6.8 & 320 \\
\hline Methane & 0.34 & 11 & & \\
\hline Acetate & & & 67.6 & 3200 \\
\hline Glycolaldehyde & & & 27.1 & 1300 \\
\hline Acetaldehyde & 0.10 & 3 & & \\
\hline Ethanol & 0.42 & 14 & & \\
\hline 1-Propanol & 1.80 & 60 & & \\
\hline
\end{tabular}

$20.5 \mathrm{~min}$. For high throughput operation, we typically perform electrolysis for a fraction of this time. For 5 min electrolysis at $3 \mathrm{~mA} / \mathrm{cm}^{2}$ or $15 \mathrm{~min}$ electrolysis at $1 \mathrm{~mA} / \mathrm{cm}^{2}$, Table I provides the detectability limit for the FE of primary $\mathrm{CO}_{2} \mathrm{RR}$ products. The sub-1\% FE limits for all typical major products, other than $\mathrm{CO}$ at $2.5 \%$ and formate at $6.8 \%$, ensure that a catalyst with any appreciable level of $\mathrm{CO}_{2} \mathrm{RR}$ activity will be successfully identified by this screening technique. The FE detection limit was estimated by dividing the detection limit of GC/HPLC (whichever was better) by the concentration of the formed product at a constant cathodic catalytic charge passed. The electrolysis charge can be increased/decreased to proportionally improve/worsen these FE detectability limits. The importance of the electrolyte recirculation and sealed headspace is also highlighted in Table I through comparison with the FE limits using traditional solution handling $\left(\mathrm{CO}_{2}\right.$ bubbling and single-pass electrolyte flow) and our GC and HPLC separations. Specifically, the detection limit is increased (worsened) in the flow-through configuration due to dilution. The dilution factor for products detected in the gas (liquid) phase is the ratio of the total volume of gas (liquid) flowed through and the volume of headspace (liquid) in the recirculation operation. For example, with $\mathrm{CO}_{2}$ bubbling at $20 \mathrm{SCCM}$ and single-pass electrolyte flow at $9.5 \mathrm{SCCM}$, the dilution factor would be 33 and 47.5 for gas and liquid phase and the correspondingly worsened detection limit is shown in columns 2 and 4 in Table I, respectively.

The catalyst screening throughput is determined by the number of electrolysis experiments per catalyst and the scheduling of the analytical measurements. The sampled electrolyte can be stored for later analysis, a scheduling flexibility that we typically utilize due to the long duration of the HPLC separation. The $10.5 \mathrm{~min}$ gas injection for the GC places the limit on sample throughput as the headspace is best analyzed immediately after sampling. Since electrolysis occurs while the GC is running, the 5 min electrolysis described in Table I leaves an ample $5.5 \mathrm{~min}$ of electrode and cell preparation between catalysts so that both electrochemistry and analytical chemistry operate with a 10.5 min period. The resulting 46 catalysts per $8 \mathrm{~h}$ operation is a very high catalyst characterization throughput for $\mathrm{CO}_{2} \mathrm{RR}$. HPLC detection can be performed on every catalyst at this daily throughput assuming round-the-clock operation, and we note that for catalyst discovery purposes analysis of the GC results can be used to eliminate the need for most HPLC measurements. While a variety of experiment design strategies can be employed for further increasing the sample throughput, the baseline 46 catalysts per day is used to compare the HT-ANEC system to previous $\mathrm{CO}_{2} \mathrm{RR}$ catalyst characterization methods in Fig. 3, where increasing analytical chemistry information corresponds to a high number of detected products and/or better detectability limits. The HT-ANEC occupies a unique space in this portfolio of techniques, providing the requisite characterization of product distribution to map trends in the $\mathrm{FE}$ of any $\mathrm{CO}_{2} \mathrm{RR}$ product at an unprecedented throughput.

While the reactor and its operation were designed to provide the excellent detectability limits of Table I with approximately 10 min experiment period, we note the broader utility of both recycling unreacted $\mathrm{CO}_{2}$ to increase reactant utilization and rapidly concentrating product streams. Separation of $\mathrm{CO}_{2} \mathrm{RR}$ products is a grand challenge in solar fuels and electrolysis design, and the recirculating flow cell with electrolyte nebulization is a design concept that may prove useful for 


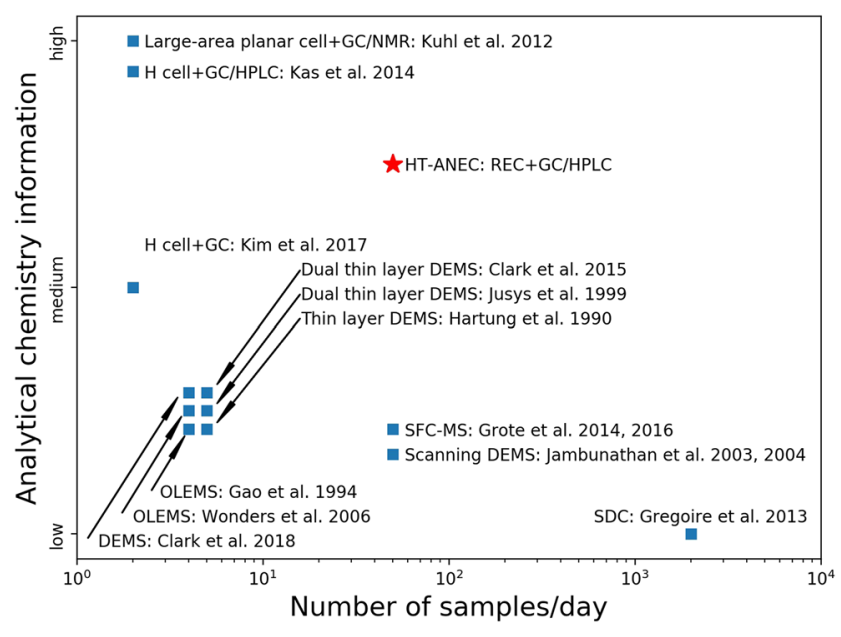

FIG. 3. Comparison between various catalyst screening techniques where the analytical chemistry information is a loosely defined combination of the variety of $\mathrm{CO}_{2} \mathrm{RR}$ products that are detectable and their respective $\mathrm{FE}$ detectability limits. The experiment throughput in terms of catalysts that can be characterized in a given workday is estimated from the duration of individual experiments for each technique and the estimated time for experiment preparation that must occur for each catalyst. The HT-ANEC systems offer a unique balance between the sample throughput and the quality of the analytical characterization of product distribution.

simultaneously optimizing mass transport for electrocatalysis and product concentration to facilitate separations.

\section{Characterizing variability in product detection}

To demonstrate the consistency of the cell operation, 6 repetitions of electrolysis (a) were performed on 6 separate samples of the as-deposited Au film. These experiments were performed on identical catalysts with a known product distribution so that any observed variations can be attributed to random sampling and separation errors. The results are shown in Fig. 4 and demonstrate excellent reproducibility with FE standard deviations of $3.8 \%$ and $2.2 \%$ for $\mathrm{CO}$ and $\mathrm{H}_{2}$, respectively. Trace $\mathrm{CH}_{4}$ was also observed and scaled by a factor of 100 for visual clarity. While the $\mathrm{CH}_{4}$ values show an outlier followed by 5 relatively consistent values, we take the

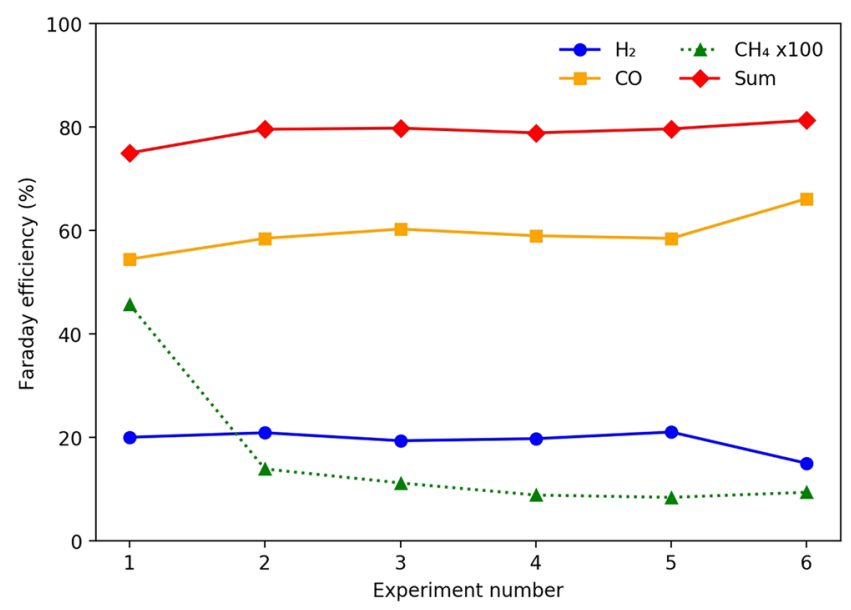

FIG. 4. Reproducibility tests of six consecutive CO2RR using duplicate Au electrodes to assess the variability in product sampling and quantification of the HT-ANEC system. standard deviation of all 6 values as the conservative estimate of the system's uncertainty. Under the assumption that the standard deviation of repeated experiments characterizes the relative error of the sampling mechanism and separation method, the absolute uncertainty in each subsequent measurement was calculated accordingly. We note that this measurement uncertainty is characteristic of the HT-ANEC system and thus does not account for any variation in catalyst preparation. In addition, equipment failure such as a septum leak can introduce additional variability in electrolysis measurements.

\section{Product distribution screening}

After validating its reproducibility, the capabilities of the HT-ANEC system were demonstrated by characterizing the $\mathrm{CO}_{2} \mathrm{RR}$ product distribution of a Pd-Au composition spread. After electrolysis, energy-dispersive X-ray spectroscopy (EDX) measurements were performed on nine spots along the same composition axis on which the electrolyses were carried out. These nine spots are designated sample numbers 1-9 (see Table II), and the EDX-determined compositions are used to characterize composition-dependent catalyst performance.

Each sample was sequentially exposed to electrolysis conditions (a)-(d), resulting in the composition-dependent product distributions of Figs. 5, 6, 7, and 8, respectively. $\mathrm{H}_{2}$ and $\mathrm{CO}$ were the dominant products; however, trace amounts of $\mathrm{CH}_{4}$ were also detected for all 4 electrolysis conditions on all samples. Given the lack of $\mathrm{CH}_{4}$ in previous studies on the Au-Pd system using similar electrochemical conditions without recirculation, ${ }^{35,37}$ we believe $\mathrm{CH}_{4}$ results from the reduction of dissolved $\mathrm{CO}$ as a consequence of its recirculation, and therefore increased concentration, through the electrolysis chamber.

The FE for $\mathrm{CO}$ production is observed to increase in the 30-35 at. \% Pd range, followed by a decrease upon further addition of Pd. Figure 9 shows the compositional variation in both uncompensated and resistance-compensated potentials ( $\mathrm{V}$ vs. RHE) for electrolysis conditions (a)-(c), revealing that the

TABLE II. List of 11 thin film catalysts in the Au-Pd system where the composition of the Au-Pd alloy catalysts was measured by EDX after electrolysis.

\begin{tabular}{lcc}
\hline \hline & \multicolumn{2}{c}{ After electrolysis } \\
\cline { 2 - 3 } Sample & Pd (at. \%) & Au (at. \%) \\
\hline 0 & 0.0 & 100.0 \\
1 & 14.9 & 85.1 \\
2 & 22.8 & 77.2 \\
3 & 34.4 & 65.7 \\
4 & 43.1 & 57.0 \\
5 & 50.6 & 49.4 \\
6 & 61.3 & 38.7 \\
7 & 69.9 & 30.1 \\
8 & 78.6 & 21.4 \\
9 & 79.8 & 20.2 \\
10 & 100.0 & 0.0 \\
\hline \hline
\end{tabular}




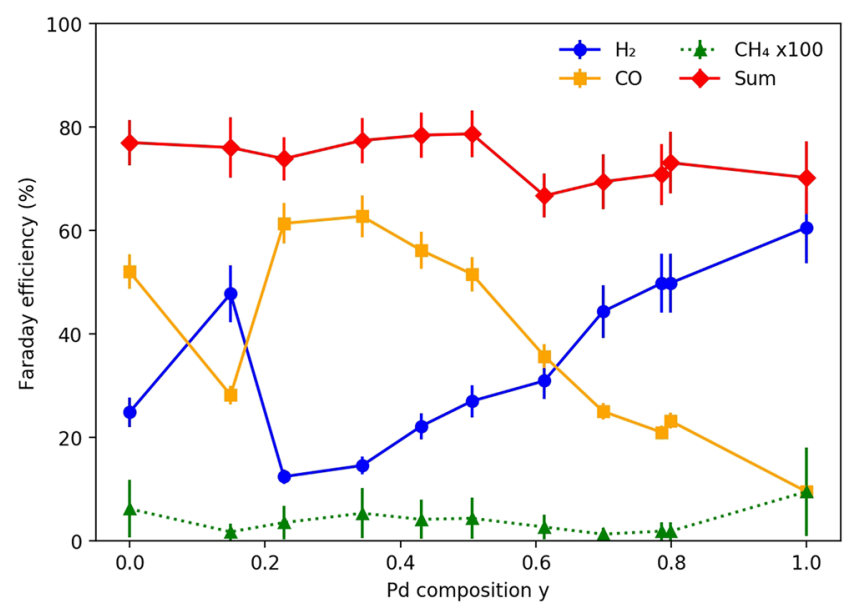

FIG. 5. Electrolysis (a). Faradaic efficiencies of $\mathrm{CO}_{2}$ reduction products of $\mathrm{Au}_{1-\mathrm{y}} \mathrm{Pd}_{\mathrm{y}}$ alloys during $15 \mathrm{~min}$ chronopotentiometric measurements at $-3 \mathrm{~mA} / \mathrm{cm}^{2}$.

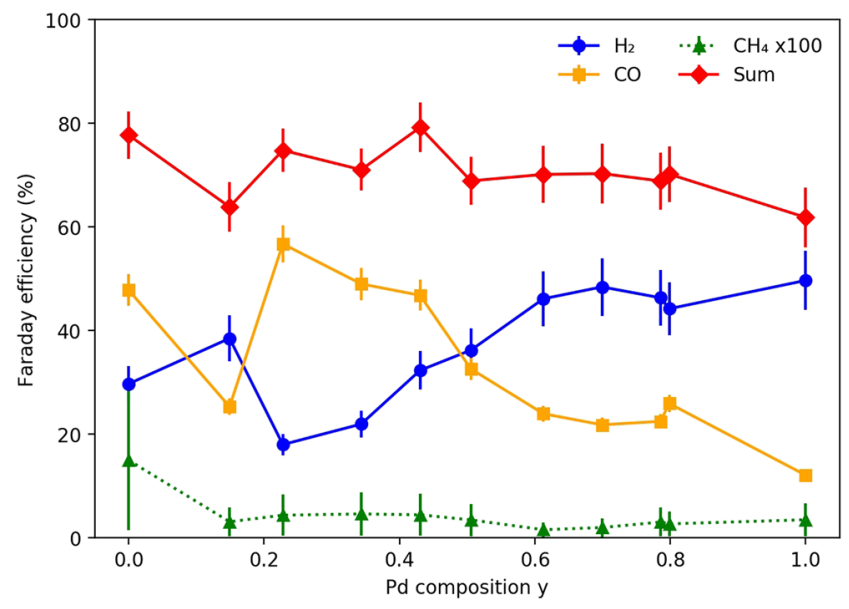

FIG. 6. Electrolysis (b). Faradaic efficiencies of $\mathrm{CO}_{2}$ reduction products of $\mathrm{Au}_{1-\mathrm{y}} \mathrm{Pd}_{\mathrm{y}}$ alloys during $15 \mathrm{~min}$ chronopotentiometric measurements at $-1 \mathrm{~mA} / \mathrm{cm}^{2}$.

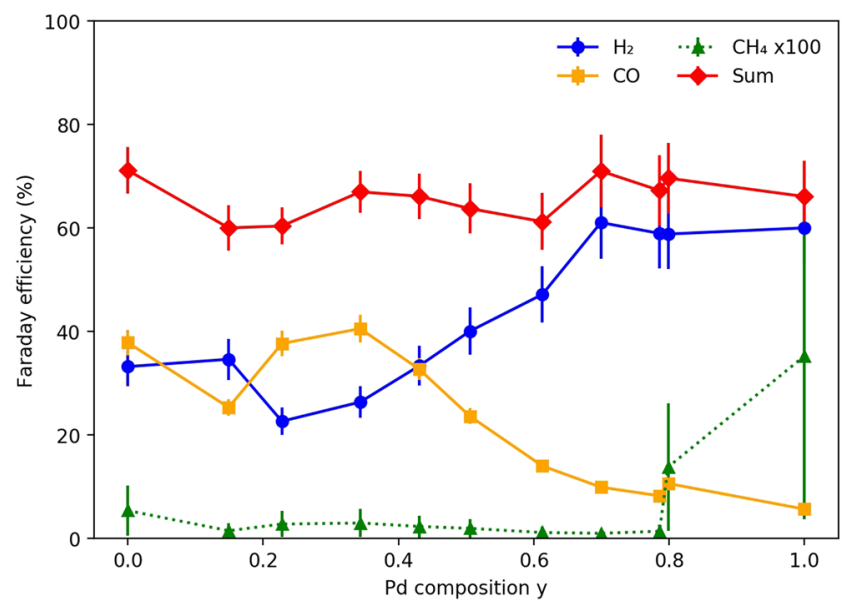

FIG. 7. Electrolysis (c). Faradaic efficiencies of $\mathrm{CO}_{2}$ reduction products of $\mathrm{Au}_{1-\mathrm{y}} \mathrm{Pd}_{\mathrm{y}}$ alloys during $4.5 \mathrm{~min}$ chronopotentiometric measurements at $-10 \mathrm{~mA} / \mathrm{cm}^{2}$.

35 at. $\% \mathrm{Pd}$ not only exhibits the highest $\mathrm{FE}$ for $\mathrm{CO}_{2} \mathrm{RR}$ but also operates at lower overpotentials for each current density compared to pure $\mathrm{Au}$ and $\mathrm{Pd}$. This specific composition range and

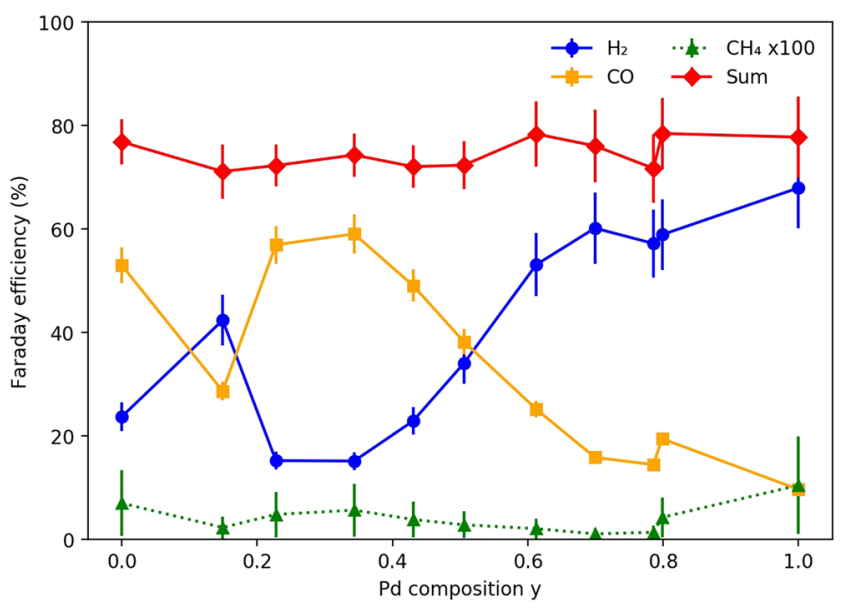

FIG. 8. Electrolysis (d), repeat of electrolysis (a). Faradaic efficiencies of $\mathrm{CO}_{2}$ reduction products of $\mathrm{Au}_{1-\mathrm{y}} \mathrm{Pd}_{\mathrm{y}}$ alloys during $15 \mathrm{~min}$ chronopotentiometric measurements at $-3 \mathrm{~mA} / \mathrm{cm}^{2}$.

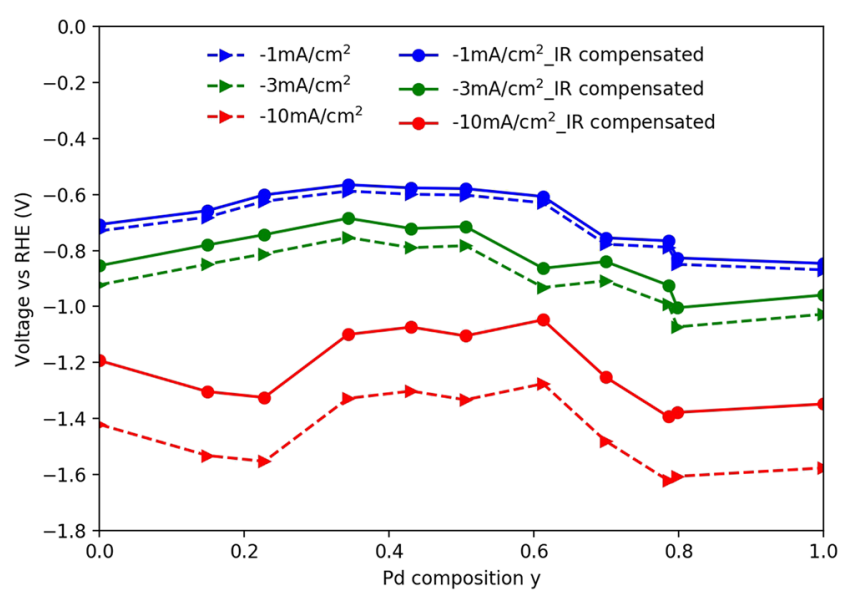

FIG. 9. Average voltage vs RHE, plotted against $\mathrm{Pd}$ composition in $\mathrm{Au}_{1-\mathrm{y}}$ $\mathrm{Pd}_{\mathrm{y}}$ alloys for each current density.

associated catalytic activity has not been reported previously, and its identification as the most efficient $\mathrm{CO}_{2} \mathrm{RR}$ catalyst in this system highlights the utility of performing combinatorial experiments. ${ }^{8-10}$ While formic acid is prominent in Pd-based reactions operating at low overpotentials, its reported FE in the potential range of the present work is on the order of $1 \%$ placing it outside of our detectable range. ${ }^{36}$

A potential cause for concern with Figs. 5-8 is the sub$100 \%$ total Faradaic efficiency. Known processes that could result in this phenomenon include product loss due to membrane crossover and parasitic reactions due to solution contaminants such as $\mathrm{O}_{2}$. To assess the possibility of product crossover, the $\mathrm{CE}$ chamber was replaced with an auxillary sampling chamber similar in geometry to the primary electrolyte recirculation chamber. Any products that permeate through the membrane from the WE chamber are thus concentrated in the auxillary sampling chamber and can be identified by the analytical instruments. Calibration gas containing $\mathrm{H}_{2}$ and $\mathrm{CO}$ (balanced with $\mathrm{CO}_{2}$ ) was introduced into the main recirculation chamber, and the REC was operated according to the prescribed electrolysis procedure but without any electrochemistry. Both primary and secondary chambers were 
sampled after $15 \mathrm{~min}$ recirculation; however, no products were observed to permeate the membrane. To assess the effect of electrolyte purity, the electrolyte was cleaned by Chelex 100 resin (Sigma-Aldrich) and a fresh Au sample was exposed to electrolysis condition (a). Comparison of the subsequent product distribution between purified and non-purified electrolytes was conducted, and the observed $5 \%$ relative increase of the total Faradaic efficiency is within the measurement uncertainty and still well below $100 \%$. It is worth noting that similar levels of total Faradaic efficiency are routinely observed using traditional methods..$^{13,35,38}$ For the stated design criteria, the most critical metric is consistency in the constituent FE measurements so that compositional trends in product selectivity and activity can be identified. Given the demonstrated consistency of our measured values, the HT-ANEC system is suitable for catalyst screening and spatial mapping for product selectivity and activity over combinatorial libraries.

Comparison of results from electrolyses (a) and (d) (both $-3 \mathrm{~mA} / \mathrm{cm}^{2}$ ) provide characterization of the evolution of the Pd-Au composition library during the course of the electrolysis experiments. Figure 10 shows the comparison, revealing no substantial change in product distribution for any of the Au-rich compositions. Pd-rich compositions have an increased $\mathrm{FE}$ for $\mathrm{H}_{2}$ and lower $\mathrm{FE}$ for $\mathrm{CO}$ in electrolysis (d) compared to electrolysis (a), indicating a degradation in the $\mathrm{CO}_{2} \mathrm{RR}$ performance. Hahn et al. ${ }^{35}$ reported Pd segregation after electrochemical tests on Au-Pd alloys, which would make Pd-rich alloys behave more like pure $\mathrm{Pd}$ as the electrolysis proceeds, which is precisely the observation from Fig. 10. These results demonstrate the utility of the HT-ANEC system in rapidly characterizing the variation in a catalysts's product distribution over time, which could also be deployed to characterize catalyst stability.

Given the limited breadth of products in the Pd-Au experiments, we provide a final demonstration of the HT-ANEC system for $\mathrm{CO}_{2} \mathrm{RR}$ using $\mathrm{Cu}$ foil (Aldrich, $99.999 \%$ metals basis, thickness $1.0 \mathrm{~mm}$ ) operated at $-1.01 \mathrm{~V}$ vs RHE with $3 \mathrm{C}$ cathodic charge passed during electrolysis. Both gaseous and liquid products were detected. A comparison was made with a $\mathrm{CO}_{2} \mathrm{RR}$ conducted under a similar voltage but

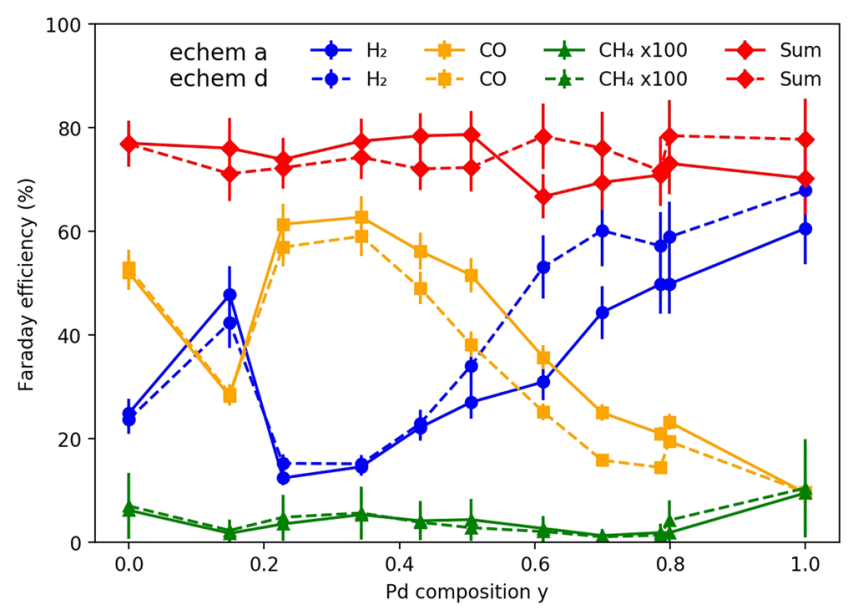

FIG. 10. Comparison between electrolyses (a) and (d): $15 \mathrm{~min} \mathrm{CP}$ at 3 $\mathrm{mA} / \mathrm{cm}^{2}$. different electrochemical cell design by Kuhl et al. ${ }^{3} \mathrm{We}$ detected the following $7 \mathrm{CO}_{2} \mathrm{RR}$ products and associated FE: CO $(32.14 \%)$, methane $(0.09 \%)$, acetaldehyde $(0.42 \%)$, ethanol $(0.28 \%)$, propionaldehyde $(0.34 \%)$, acetone $(0.04 \%)$, and formate $(4.83 \%)$, which are qualitatively in agreement with Kuhl $e t \mathrm{al}^{3}{ }^{3}$ The primary quantitative differences are higher $\mathrm{CO}$ and lower ethanol FE in our experiments, and associated with the low ethanol FE is the absence of ethylene in our experiment. These results show the ability of the HT-ANEC system to rapidly identify a broad range of gaseous and liquid products even with sub-1\% FE and indicate a sensitivity (with respect to cell and flow parameters) of reaction products from $\mathrm{Cu}$, which is commensurate with the range of $\mathrm{FE}$ values reported in the literature. We note that a composition library with variation in production of high-order hydrocarbons and alcohols would provide an even more compelling demonstration of HT-ANEC, but the lack of known catalyst systems of this type is precisely the motivation for developing the instrument.

\section{SUMMARY}

A recirculating electrochemical reactor cell (REC) is presented and optimized for catalyst screening in conditions relevant to $\mathrm{CO}_{2} \mathrm{RR}$ where rapid electrolyte flow facilitates mass transport at the catalyst surface, and electrolyte recirculation with nebulization in a sealed headspace enables rapid concentration of products to facilitate their detection and/or separation. By integrating the REC reactor with an automatic sampling mechanism and analytical instruments, the high throughput analytical electrochemistry (HT-ANEC) system enables an unprecedented combination of product detection and experiment throughput for $\mathrm{CO}_{2} \mathrm{RR}$ catalyst screening with a FE detectability limit of $\sim 1 \%$ for most $\mathrm{CO}_{2} \mathrm{RR}$ products and an experimental duration of approximately $10 \mathrm{~min}$. The capabilities of the HT-ANEC system were demonstrated with $\mathrm{CO}_{2} \mathrm{RR}$ screening of Au-Pd alloys, resulting in identification of the $\mathrm{Au}_{0.7} \mathrm{Pd}_{0.3}$ composition as both the most active and selective for $\mathrm{CO}_{2}$ reduction to $\mathrm{CO}$. The HT-ANEC features similar time scales for electrochemical methods and analytical techniques to mitigate bottlenecks and maximize information throughput, and its successful deployment and full automation will further facilitate the exploration of catalysts for $\mathrm{CO}_{2} \mathrm{R}$ and other reactions where mapping product selectivity is central to catalyst discovery.

\section{ACKNOWLEDGMENTS}

This material is based upon work performed by the Joint Center for Artificial Photosynthesis, a DOE Energy Innovation Hub, supported through the Office of Science of the U.S. Department of Energy under Award No. DE-SC0004993. We thank Dr. Lan Zhou for providing the Pd-Au composition spread library.

\footnotetext{
${ }^{1}$ A. Fujishima and K. Honda, Nature 238, 37 (1972).

${ }^{2}$ L. Pan, J. Hyun Kim, M. T. Mayer, M.-K. Son, A. Ummadisingu, J. Sung Lee, A. Hagfeldt, J. Luo, and M. Gramp, Nat. Catal. 1, 412 (2018).

${ }^{3}$ K. P. Kuhl, E. R. Cave, D. N. Abram, and T. F. Jaramillo, Energy Environ. Sci. 5, 7050 (2012)
} 
${ }^{4}$ C. Delacourt, P. L. Ridgway, J. B. Kerr, and J. Newman, J. Electrochem. Soc. 155, B42 (2008).

${ }^{5}$ C. Liang, B. Kim, S. Yang, Y. Liu, C. Woellner, Z. Li, R. Vajtai, W. Yang, J. Wu, P. J. A. Kenis, and P. M. Ajayan, J. Mater. Chem. A 6, 10313 (2018). ${ }^{6}$ E. E. Barton, D. M. Rampulla, and A. B. Bocarsly, J. Am. Chem. Soc. 130, 6342 (2008).

${ }^{7}$ T. Arai, S. Sato, K. Uemura, T. Morikawa, T. Kajino, and T. Motohiro, Chem. Commun. 46, 6944 (2010).

${ }^{8}$ R. Potyrailo, K. Rajan, K. Stowe, I. Takeuchi, B. Chisholm, and H. Lam, ACS Comb. Sci. 13, 579 (2011).

${ }^{9}$ H. Zhang, R. Hoogenboom, M. A. R. Meier, and U. S. Schubert, Meas. Sci. Technol. 16, 203 (2005).

${ }^{10}$ R. A. Potyrailo and V. M. Mirsky, Chem. Rev. 108, 770 (2008).

${ }^{11}$ W. Zhu, R. Michalsky, Ö. Metin, H. Lv, S. Guo, C. Wright, X. Sun, A. A. Peterson, and S. Sun, J. Am. Chem. Soc. 139, 9408 (2017).

${ }^{12}$ H. Kim, H. S. Park, Y. J. Hwang, and B. K. Min, J. Phys. Chem. C 121, 22637 (2017)

${ }^{13}$ Y. Peng, T. Wu, L. Sun, J. M. V. Nsanzimana, A. C. Fisher, and X. Wang, ACS Appl. Mater. Interfaces 9, 32782 (2017).

${ }^{14}$ H. Noda, S. Ikeda, Y. Oda, K. Imai, M. Maeda, K. Ito, S. Ideka, Y. Oda, K. Imai, M. Maeda, and I. Kaname, Bull. Chem. Soc. Jpn. 63, 2459 (1990).

${ }^{15}$ N. E. Şahin, C. Comminges, A. LeValant, J. Kiener, J. Parmentier, T. W. Napporn, G. Melinte, O. Ersen, and K. B. Kokoh, ChemPhysChem 19, 1371 (2018).

${ }^{16}$ S. Rasul, A. Pugnant, and E. H. Yu, ECS Trans. 85, 57 (2018).

${ }^{17}$ S. Lee, G. Park, and J. Lee, ACS Catal. 7, 8594 (2017).

${ }^{18}$ H. Baltruschat, J. Am. Soc. Mass Spectrom. 15, 1693 (2004).

${ }^{19}$ S. J. Ashton, Springer Theses 8, 9 (2012).

${ }^{20}$ E. L. Clark and A. T. Bell, J. Am. Chem. Soc. 140, 7012 (2018).

${ }^{21}$ O. Wolter and J. Heitbaum, Ber. Bunsenges. Phys. Chem. 88, 2 (1984).

${ }^{22} \mathrm{~T}$. Hartung and H. Baltruschat, Langmuir 6, 953 (1990).
${ }^{23}$ Z. Jusys, J. Electrochem. Soc. 146, 1093 (1999).

${ }^{24}$ Y. Gao, H. Tsuji, H. Hattori, and H. Kita, J. Electroanal. Chem. 372, 195 (1994).

${ }^{25}$ A. H. Wonders, T. H. M. Housmans, V. Rosca, and M. T. M. Koper, J. Appl. Electrochem. 36, 1215 (2006).

${ }^{26}$ K. Jambunathan and A. C. Hillier, J. Electrochem. Soc. 150, E312 (2003).

${ }^{27}$ K. Jambunathan, S. Jayaraman, and A. C. Hillier, Langmuir 20, 1856 (2004).

${ }^{28}$ J. M. Gregoire, C. Xiang, X. Liu, M. Marcin, and J. Jin, Rev. Sci. Instrum. 84, 024102 (2013).

${ }^{29}$ J. P. Grote, A. R. Zeradjanin, S. Cherevko, A. Savan, B. Breitbach, A. Ludwig, and K. J. J. Mayrhofer, J. Catal. 343, 248 (2016).

${ }^{30}$ S. O. Klemm, A. A. Topalov, C. A. Laska, and K. J. J. Mayrhofer, Electrochem. Commun. 13, 1533 (2011).

${ }^{31}$ J. P. Grote, A. R. Zeradjanin, S. Cherevko, and K. J. J. Mayrhofer, Rev. Sci. Instrum. 85, 104101 (2014).

${ }^{32}$ E. L. Clark, M. R. Singh, Y. Kwon, and A. T. Bell, Anal. Chem. 87, 8013 (2015).

${ }^{33}$ S. K. Suram, L. Zhou, N. Becerra-Stasiewicz, K. Kan, R. J. R. Jones, B. M. Kendrick, and J. M. Gregoire, Rev. Sci. Instrum. 86, 033904 (2015).

${ }^{34}$ M. R. Thorson, K. I. Siil, and P. J. A. Kenis, J. Electrochem. Soc. 160, F69 (2012).

${ }^{35}$ C. Hahn, D. N. Abram, H. A. Hansen, T. Hatsukade, A. Jackson, N. C. Johnson, T. R. Hellstern, K. P. Kuhl, E. R. Cave, J. T. Feaster, and T. F. Jaramillo, J. Mater. Chem. A 3, 20185 (2015).

${ }^{36}$ D. M. Weekes, D. A. Salvatore, A. Reyes, A. Huang, and C. P. Berlinguette, Acc. Chem. Res. 51, 910 (2018).

${ }^{37}$ R. Kortlever, I. Peters, C. Balemans, R. Kas, Y. Kwon, G. Mul, and M. T. M. Koper, Chem. Commun. 52, 10229 (2016).

${ }^{38}$ R. Kas, R. Kortlever, A. Milbrat, M. T. M. Koper, G. Mul, and J. Baltrusaitis, Phys. Chem. Chem. Phys. 16, 12194 (2014). 